\title{
]jfis
}

\section{Study of Nonlinear Fuzzy Integro-differential Equations Using Mathematical Methods and Applications}

\author{
Jamshad Ahmad ${ }^{1}$, Angbeen Iqbal ${ }^{1}$, and Qazi Mahmood UI Hassan ${ }^{2}$ \\ ${ }^{1}$ Department of Mathematics, Faculty of Science, University of Gujrat, Punjab, Pakistan \\ ${ }^{2}$ Department of Mathematics, University of Wah, Punjab, Pakistan
}

Received: Dec. 11, 2020

Revised : Feb. 1, 2021

Accepted: Feb. 22, 2021

Correspondence to: Jamshad Ahmad (jamshadahmadm@gmail.com)

(CThe Korean Institute of Intelligent Systems

(c)This is an Open Access article distributed under the terms of the Creative Commons Attribution Non-Commercial License (http://creativecommons.org/licenses/by-nc / 3.0/ which permits unrestricted noncommercial use, distribution, and reproduction in any medium, provided the original work is properly cited.

\begin{abstract}
In this study, the homotopy perturbation sumudu transform method (HPSTM) is employed to find the analytical fuzzy solution of nonlinear fuzzy integro-differential equations (FIDEs). The solutions of FIDEs are more generalized and have better applications. The fuzzy concept is used to overrule the uncertainty in physical models. Based on the parametric form of the fuzzy number, the nonlinear integro-differential equation (IDE) is converted into two systems of nonlinear IDEs of the second kind. Some numerical examples were solved to demonstrate the efficiency and capability of the method. Graphical representations reveal the symmetry between lower and upper cut representations of fuzzy solutions and may be helpful for a better understanding of fuzzy control models, artificial intelligence, medical science, quantum optics, measure theory, and so on.
\end{abstract}

Keywords: Sumudu transform, Homotopy perturbation method, Non linear fuzzy integro-differential equation, Fuzzy solution

\section{Introduction}

The notions of fuzzy differential equations and fuzzy integral equations with fuzzy control have attracted researchers. Different definitions of fuzzy derivatives and fuzzy integrals have been established and extended to fuzzy calculus. For the existence and uniqueness of the solution of these equations, sufficient conditions were provided, and different numerical algorithms were designed for the approximate solution. The fuzzy integral equation theory is new, and plays a key role in various fields of engineering, applied mathematics, physics, biological sciences, optimal control theory, mathematical economics, etc. Modeling any physical problem by using integral equations with exact parameters is not an easy task; in real-world problems, it is almost impossible. Thus, it is nearly impossible to deal with exact parameters in real-life problems. Therefore, many researchers have worked on these models to investigate the solutions of fuzzy integral equations. To overcome this problem, a reliable approach is to use the fuzzy concept. Elementary idea of "fuzzy set" by Zadeh [1, 2] in his publications of fuzzy set theory. In 1982, Dubois and Prade [3] presented arithmetic operations on fuzzy calculus. The study of fuzzy integral and differential equations has been rapidly advancing in recent years [4]-8]. In recent years, research on fuzzy differential equations and fuzzy integral equations from both theoretical and numerical points of view have been developed. Analytical solutions of some 
fuzzy integro-differential equations (FIDEs) using the analytical method, Laplace homotopy perturbation method (HPM), were produced [9]. Hamoud and Ghadle [10] provided a solution for fuzzy Volterra integro-differential equation (IDE) via modified Adomian decomposition method. Hooshangian [11] proposed a solution for the fuzzy Volterra IDE of the $n$-th order with a nonlinear fuzzy kernel and converted it into a nonlinear integral equation by using the generalized Hakuhara derivative. Therefore, finding an efficient and accurate algorithm for investigating fuzzy integral equation has recently garnered significant attention among researchers. The existence and uniqueness of the solution of the nonlinear FIDE and impulsive semi-linear FIDEs with nonlocal conditions and forcing the term with memory is well addressed in [12, 13]. Recently, Padmapriya et al. [14] investigated the numerical solutions of fuzzy fractional delay differential equations using the proposed novel technique. The HPM was first proposed by He [15, 16], who also approximated the answers for several differential equations. This method is a merge of homotopy defined in topology with the traditional perturbation method. The author has practiced HPM on a wide range of applications in computing functional equations [17]. The Sumudu transform was first introduced by Watagula in [18]. The homotopy perturbation Sumudu transform method (HPSTM) is the coupling of the Sumudu transform and HPM. The proposed method has advantages such as a simple structure, ease of programming, and high accuracy. Motivated by the aforementioned work, in this study, we use a HPSTM to solve the nonlinear FIDE. The remainder of this paper is organized as follows. In Section 2, we introduce some preliminaries and basic definitions. In Section 3, we present the proposed algorithm. To demonstrate the effectiveness of the proposed method, we present some numerical results on several tests in Section 4 . Finally, some concluding remarks are presented in Section 5.

\section{Preliminaries}

Definition 2.1. The Sumudu transform of any set B is defined as

$$
B=\left\{v: v(t)<M e^{\frac{|t|}{k}}, t \epsilon(-1)^{j} \times[0, \infty),\left(M, k_{1}, k_{2}>0\right)\right\}
$$

The Sumudu transform method [18] is written as follows:

$$
S(f(t))=\int_{0}^{\infty} f(u t) e^{-t}=F(u), u \epsilon\left(-k_{1}, k_{2}\right) .
$$

Definition 2.2. The general form of the FIDE is as follows:

$$
y^{(m)}(t, \beta)=g(t, \beta)+\lambda \int_{a(t)}^{b(t)} k(t, s) F(y(s, \beta)) d s .
$$

$y(0, \beta)=\left(a_{0}, b_{0}\right), y^{\prime}(0, \beta)=\left(a_{1}, b_{1}\right), y^{\prime \prime}(0, \beta)=\left(a_{2}, b_{2}\right)^{\prime}$, $\ldots, y^{m-1}(0, \beta)=\left(a_{m-1}, b_{m-1}\right)$, where $\left(a_{j}, b_{j}\right)$ with $j=$ $0,1,2,3, \ldots m-1$ are known to remain constant. $y^{m}(t, \beta)$ is the $m^{t h}$ order derivative of the fuzzy function and is already given, $\beta$ is a fuzzy parameter with values between $[0,1](0 \leq$ $\beta \leq 1), \lambda$ is known to be a constant parameter, and $\mathrm{K}(\mathrm{t}, \mathrm{s})$ is the kernel of this FIDE and depends on variable $t$ and s. $a(t)$ and $b(t)$ are known to be the limits of this FIDE. If these limits are constant, the IDE will be called a fuzzy-Fredholm IDE, and if one of these limits is variable, then this equation will be said fuzzy-Volterra IDE:

In parametric form Eq. (3) is represented as

$$
\left\{\begin{array}{l}
\underline{y}^{(m)}(t, \beta)=\underline{g}(t, \beta)+\lambda \int_{a(t)}^{b(t)} \frac{k(t, s) F(y(s, \beta))}{b(t)} d s, \\
\bar{y}^{(m)}(t, \beta)=\bar{g}(t, \beta)+\lambda \int_{a(t)} \overline{k(t, s) F(y(s, \beta))} d s .
\end{array}\right.
$$

In addition, $0 \leq \beta \leq 1$ and $F(y(s, \beta))=(\underline{F}(y(s, \beta)), \bar{F}(y(s, \beta)))$, $g(t, \beta)=(\underline{g}(t, \beta), \bar{g}(t, \beta))$, with kernel

$$
\left\{\begin{array}{l}
\frac{K(t, s) F(y(s, \beta))}{\overline{K(t, s) F(y(s, \beta))}}=K(t, s) \underline{F}(y(s, \beta)), \quad(t, s) \geq 0, \\
\bar{F}(y(s, \beta)), K(t, s) \leq 0 .
\end{array}\right.
$$

\section{Methodology Description}

To illustrate the basic representation of the HPSTM on the FIDE in general form, the basic fuzzy condition is defined in Eq. (3)

$$
\left\{\begin{array}{l}
H(\underline{x}, p, \beta) \\
=(1-p)\left[\underline{x}^{(m)}(t, \beta)-\underline{y}_{0}(t, \beta)\right] \\
+p\left[\underline{x}^{(m)}(t, \beta)-\underline{g}(t, \beta)-\int_{a(t)} k(t, s) \underline{F}(x(s, \beta)) d s\right]=0, \\
H(\bar{x}, p, \beta) \\
=(1-p)\left[\bar{x}^{(m)}(t, \beta)-\bar{y}_{o}(t, \beta)\right] \\
+p\left[\bar{x}^{(m)}(t, \beta)-\bar{g}(t, \beta)-\int_{a(t)}^{k(t)} k(t, s) \bar{F}(x(s, \beta))\right]=0 .
\end{array}\right.
$$

And the initial approximation is reserved as 


$$
\left\{\begin{array}{l}
\underline{x}_{0}(t, \beta)=\underline{x}(t, \beta), \\
\bar{x}_{0}(t, \beta)=\bar{g}(t, \beta) .
\end{array}\right.
$$

Substituting Eq.(4) in Eq.(3) as

$$
\left\{\begin{array}{l}
\underline{x}^{(m)}(t, \beta)=\underline{g}(t, \beta)+p \int_{a(t)}^{b(t)} k(t, s) \underline{F}(x(s, \beta)) d s, \\
\bar{x}^{(m)}(t, \beta)=\bar{g}(t, \beta)+p \int_{a(t)}^{b(t)} k(t, s) \bar{F}(x(s, \beta)) d s .
\end{array}\right.
$$

Applying Sumudu transform on both sides of Eq. (7) as

$$
\left\{\begin{array}{l}
S\left\{\underline{x}^{(m)}(t, \beta)\right\} \\
=S\left\{\underline{g}(t, \beta)+p \int_{a(t)}^{b(t)} k(t, s) \underline{F}(x(s, \beta)) d s\right\}, \\
S\left\{\bar{x}^{(m)}(t, \beta)\right\} \\
=S\left\{\bar{g}(t, \beta)+p \int_{a(t)}^{b(t)} k(u, v) \bar{F}(x(s, \beta)) d s\right\} .
\end{array}\right.
$$

Applying the differential property of Sumudu transform on both sides of Eq. (8).

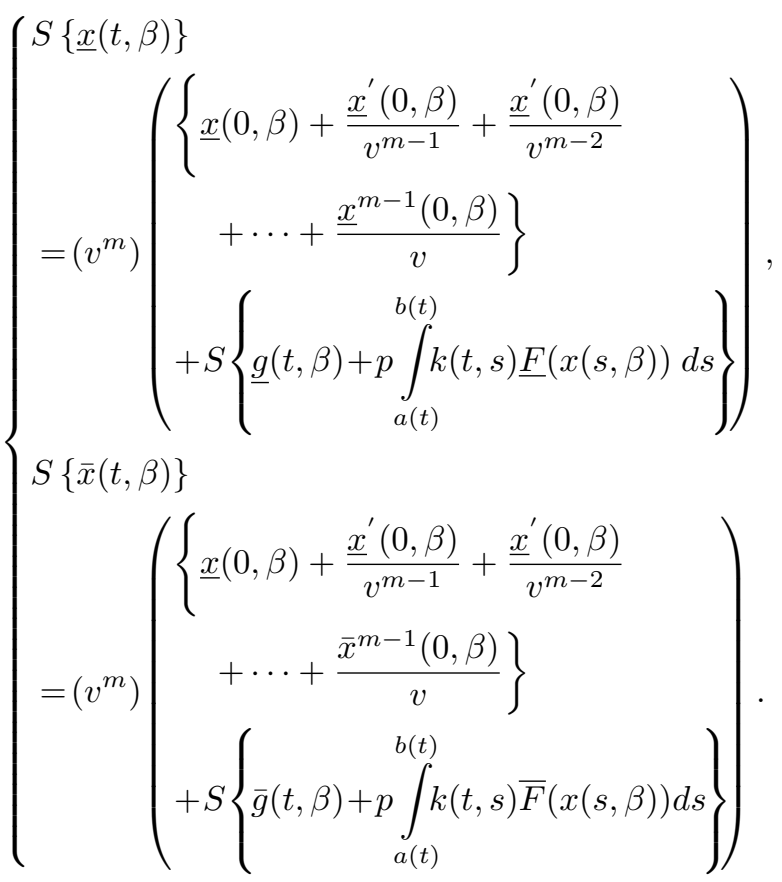

The inverse transform on each side of Eq. (9) gives

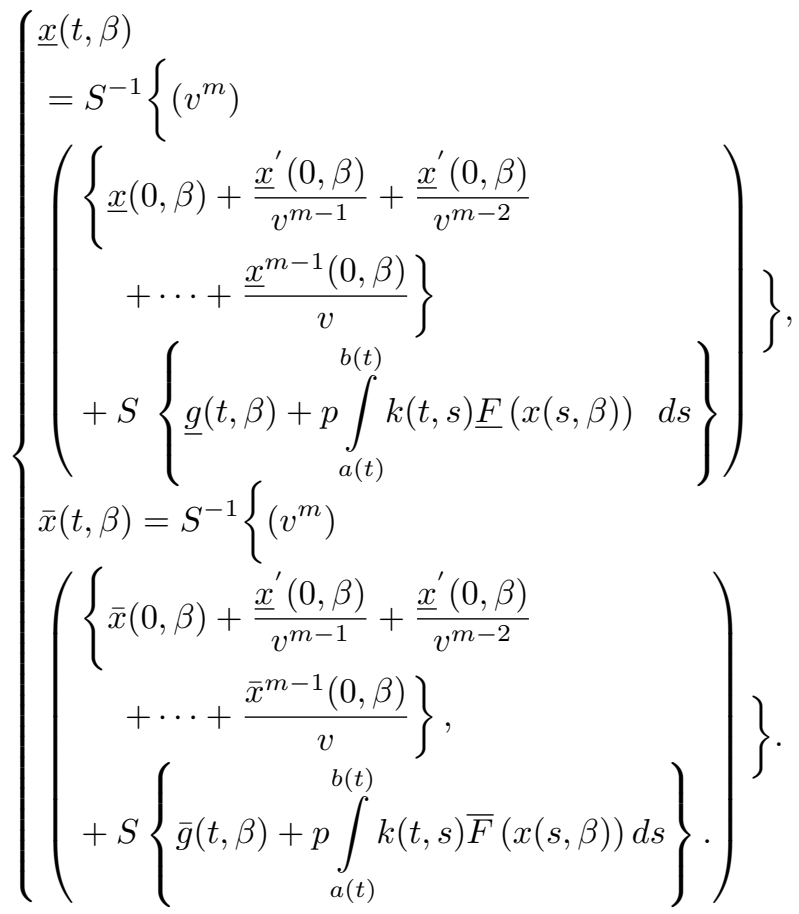

Assuming the solution of Eq. (10) is stated in power series of $p$

$$
\left\{\begin{array}{l}
\underline{x}(t, \beta)=\sum_{j=0}^{\infty} p^{j} \underline{x}_{j}, \\
\bar{x}(t, \beta)=\sum_{j=0}^{\infty} p^{j} \bar{x}_{j} .
\end{array}\right.
$$

By replacing the solution of Eq. (11) in Eq. (10) and comparing the coefficients as power of $p$, we obtain the iterations as follows:

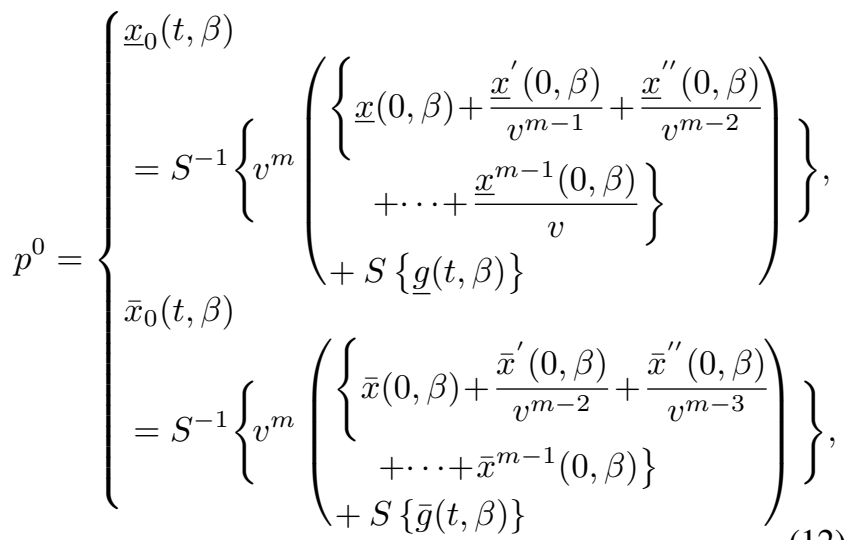




$$
p^{1}=\left\{\begin{array}{l}
\underline{x}_{1}(t, \beta) \\
=S^{-1}\left\{v^{m}\left(S\left\{p \int_{a(t)}^{b(t)} k(t, s) \underline{F}\left(x_{0}(s, \beta)\right) d s\right\}\right)\right\} \\
\bar{x}_{1}(t, \beta) \\
=S^{-1}\left\{v^{m}\left(S\left\{p \int_{a(t)}^{b(t)} k(t, s) \bar{F}\left(x_{0}(s, \beta)\right) d s\right\}\right)\right\} \\
=S^{-1}\left\{\left(v^{m}\right)\left(S\left\{p \int_{a(t)}^{b(t)} k(t, s) \underline{F}\left(x_{1}(s, \beta)\right) d s\right\}\right)\right\} \\
\bar{x}_{2}(t, \beta) \\
=S^{-1}\left\{\left(v^{m}\right)\left(S\left\{p \int_{(14}^{b(t)} k(t, s) \underline{F}\left(x_{1}(s, \beta)\right) d s\right\}\right)\right\}
\end{array}\right.
$$

and so on. Finally, the solution of FIDE-2 is given as

$$
\left\{\begin{array}{l}
\underline{y}(t, \beta)=\lim _{p \rightarrow 1} \underline{x}(t, \beta)=\sum_{j=0}^{\infty} \underline{x}_{j}(t, \beta) \\
\bar{y}(t, \beta)=\lim _{p \rightarrow 1} \bar{x}(t, \beta)=\sum_{j=0}^{\infty} \bar{x}_{j}(t, \beta)
\end{array}\right.
$$

\section{Numerical Applications}

Problem 4.1. Consider the nonlinear fuzzy Fredholm IDE of the second kind as [19]

$$
y^{\prime}(t, \beta)=g(t, \beta)+\int_{0}^{1} \frac{t^{2} s}{10} y^{2}(s, \beta) d s,
$$

with initial condition $y(0, \beta)=(0,0), \lambda=1,0 \leq s \leq 1,0 \leq$ $\beta \leq 1, K(t, s)=\frac{t^{2} s}{10}, g(t, \beta)=\left(\beta-\frac{t^{2} \beta^{2}}{40},(2-\beta)-\frac{t^{2}(2-\beta)^{2}}{40}\right)$.

The exact solution of Eq. (16) is given as $y(t, \beta)=(t \beta$, $(2-\beta))$.

The homotopy is

$$
\left\{\begin{aligned}
H(\underline{x}, p, \beta)= & \underline{x}^{\prime}(t, \beta)-\left(\beta-\frac{t^{2} \beta^{2}}{40}\right) \\
& -p \int_{0}^{1} \frac{t^{2} s}{10} \underline{x}^{2}(s, \beta) d s=0 \\
H(\bar{x}, p, \beta)= & \bar{x}^{\prime}(t, \beta)-\left((2-\beta)-\frac{t^{2}(2-\beta)^{2}}{40}\right) \\
& -p \int_{0}^{1} \frac{t^{2} s}{10} \bar{x}^{2}(s, \beta) d s=0 .
\end{aligned}\right.
$$

According to the procedure described above, we have

$$
\left\{\begin{array}{l}
\underline{x}(t, \beta) \\
=S^{-1}\left\{v\left\{S\left\{\left(\beta-\frac{t^{2} \beta^{2}}{40}\right)+p \int_{0}^{1} \frac{t^{2} s}{10} \underline{x}^{2}(s, \beta) d s\right\}\right\}\right\} \\
\bar{x}(t, \beta) \\
=S^{-1}\left\{v \left\{S \left\{\left((2-\beta)-\frac{t^{2}(2-\beta)^{2}}{40}\right)\right.\right.\right. \\
\left.\left.\left.+p \int_{0}^{1} \frac{t^{2} s}{10} \bar{x}^{2}(s, \beta) d s\right\}\right\}\right\} .
\end{array}\right.
$$

Consequently, we have

$p^{0}:\left\{\begin{array}{l}\underline{x}_{0}(t, \beta)=t \beta-\frac{t^{3} \beta^{2}}{120}, \\ \bar{x}_{0}(t, \beta)=t(2-\beta)-\frac{t^{3}(2-\beta)^{2}}{120},\end{array}\right.$

$p^{1}:\left\{\begin{array}{l}\underline{x}_{1}(t, \beta)=\frac{t^{3}}{3 !}\left(\frac{\beta^{2}}{20}+\frac{\beta^{4}}{576000}-\frac{\beta^{3}}{3600}\right), \\ \bar{x}_{1}(t, \beta)=\frac{t^{3}}{3 !}\left(\frac{(2-\beta)^{2}}{20}+\frac{(2-\beta)^{4}}{576000}-\frac{(2-\beta)^{3}}{3600}\right),\end{array}\right.$

and so on. The series solution (Figure 1) is

$$
\left\{\begin{aligned}
\underline{y}(t, \beta)= & t \beta-\frac{t^{3} \beta^{2}}{120}+\frac{t^{3} \beta^{2}}{120}+\frac{t^{3} \beta^{4}}{345600}-\frac{t^{3} \beta^{3}}{21600}+\cdots \\
\bar{y}(t, \beta)= & t(2-\beta)-\frac{t^{3}(2-\beta)^{2}}{120}+\frac{t^{3}(2-\beta)^{2}}{120} \\
& +\frac{t^{3}(2-\beta)^{4}}{345600}-\frac{t^{3}(2-\beta)^{3}}{21600}+\cdots .
\end{aligned}\right.
$$

Problem 4.2. Consider the nonlinear fuzzy Volterra IDE of the second kind as [9]

$$
y^{\prime}(t, \beta)=(\beta,(7-\beta))+\int_{0}^{t} y^{2}(s, \beta) d s,
$$

where $y(0, \beta)=(0,0), 0 \leq s \leq 1,0 \leq \alpha \leq 1, \lambda=1$, $K(t, s)=1, g(t, \beta)=(\beta,(7-\beta))$. According to the procedure described above, we have

$$
\left\{\begin{array}{c}
\underline{x}(t, \beta)=S^{-1}\left\{v\left\{S\left\{\beta+p \int_{0}^{t} \underline{x}^{2}(s, \beta) d s\right\}\right\}\right\}, \\
\bar{x}(t, \beta)=S^{-1}\left\{v\left\{S\left\{(7-\beta)+p \int_{0}^{t} \bar{x}^{2}(s, \beta) d s\right\}\right\}\right\},
\end{array}\right.
$$




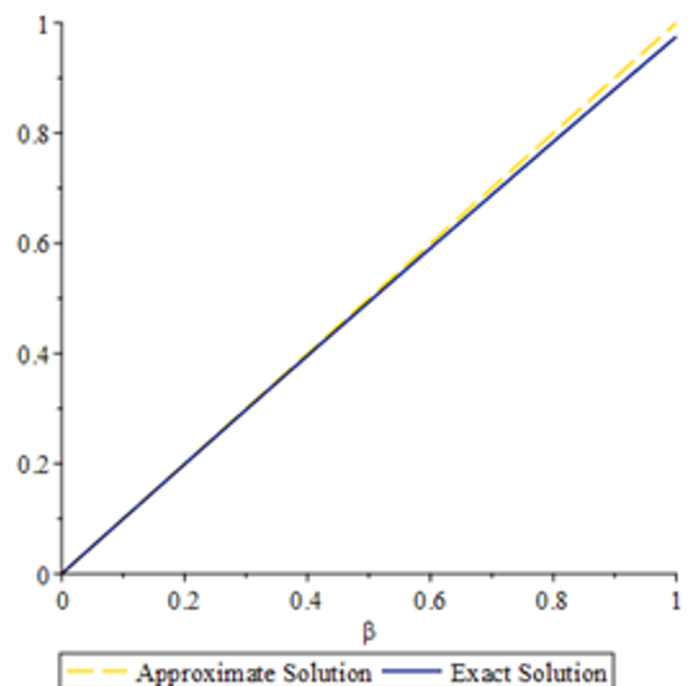

(a)

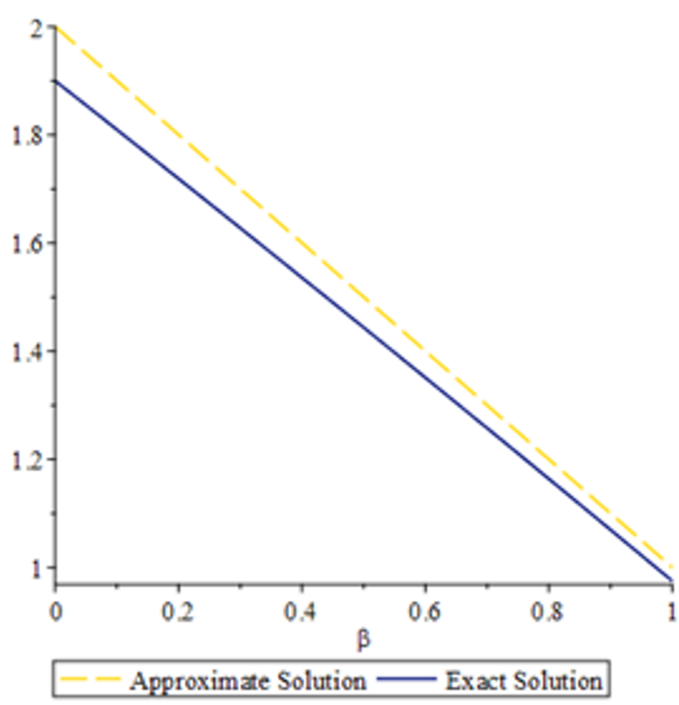

(b)

Figure 1. Comparison of approximate solution (21) and exact solution of (a) $\underline{y}$ and (b) $\bar{y}$ at $t=1$.

$$
\begin{aligned}
& p^{0}:\left\{\begin{array}{l}
\underline{x}_{0}(t, \beta)=t \beta, \\
\bar{x}_{0}(t, \beta)=t(7-\beta),
\end{array}\right. \\
& p^{1}:\left\{\begin{array}{l}
\underline{x}_{1}(t, \beta)=\beta^{2} \frac{t^{4}}{12}, \\
\bar{x}_{1}(t, \beta)=(7-\beta)^{2} \frac{t^{4}}{12},
\end{array}\right. \\
& p^{2}:\left\{\begin{array}{l}
\underline{x}_{2}(t, \beta)=\beta^{4} \frac{t^{10}}{12960}, \\
\bar{x}_{2}(t, \beta)=(7-\beta)^{4} \frac{t^{10}}{12960},
\end{array}\right.
\end{aligned}
$$

and so on. The solution (Figure 2) is given as

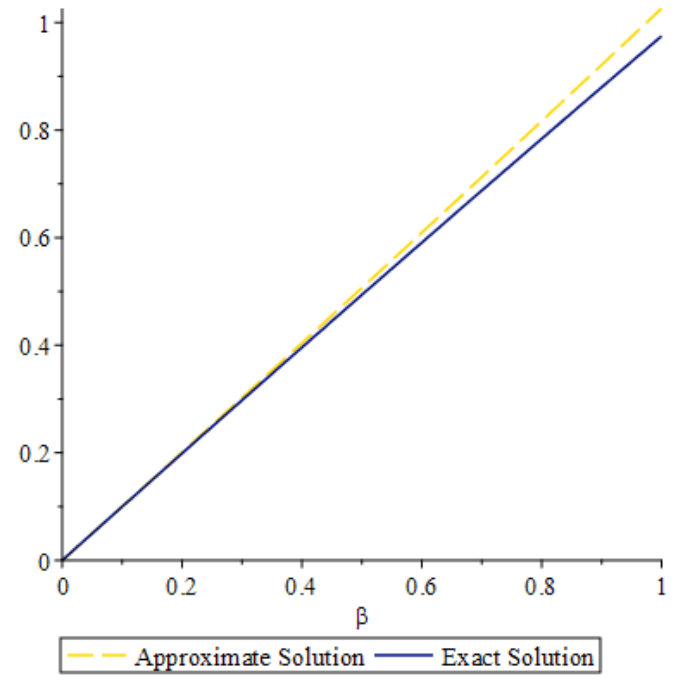

(a)

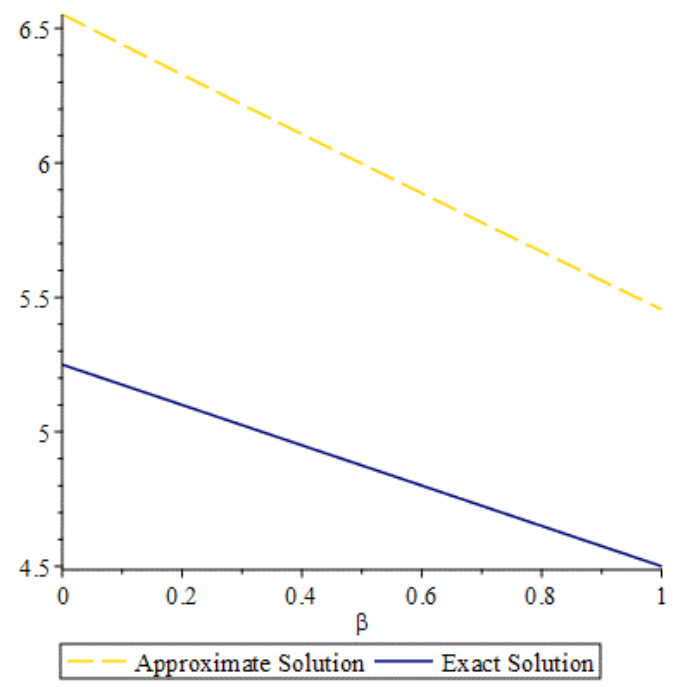

(b)

Figure 2. Comparison of approximate solution 27] and exact solution of $\underline{y}$ and (a) $\bar{y}$ at (b) $t=1$.

$$
\left\{\begin{array}{l}
\underline{y}(t, \beta)=t \beta+\beta^{2} \frac{t^{4}}{12}+\beta^{4} \frac{t^{10}}{12960}+\cdots \\
\bar{y}(t, \beta)=t(7-\beta)+(7-\beta)^{2} \frac{t^{4}}{12}+(7-\beta)^{4} \frac{t^{10}}{12960}+\cdots
\end{array}\right.
$$

Problem 4.3. Consider the nonlinear fuzzy Volterra IDE of 2nd second-order as [11]

$$
y^{\prime \prime}(t, \beta)=g(t, \beta)+\int_{0}^{t} y^{2}(s, \beta) d s,
$$


with initial values $y(0, \beta)=(\beta-1,1-\beta), y^{\prime}(0, \beta)=(\beta$, $2-\beta)$, where $g(t, \beta)=\left(-\beta^{2} t^{3},-(2-\beta)^{2} t^{3}\right), 0 \leq s \leq t$, $0 \leq \beta \leq t, \lambda=1, K(t, s)=1$.

The exact solution is given by $y(0, \beta)=(3 t \beta, 3 t(2-\beta))$. The homotopy as

$$
\left\{\begin{array}{l}
H(\underline{x}, p, \beta)=\underline{x}^{\prime \prime}(t, \beta)+\beta^{2} t^{3}-p \int_{0}^{t} \underline{x}^{2}(s, \beta) d s=0, \\
H(\bar{x}, p, \beta)=\bar{x}^{\prime \prime}(t, \beta)+(2-\beta)^{2} t^{3}-p \int_{0}^{t} \bar{x}^{2}(s, \beta) d s=0,
\end{array}\right.
$$

According to the procedure described above, we have

$$
\left\{\begin{array}{l}
\underline{x}(t, \beta) \\
=S^{-1}\left\{v ^ { 2 } \left(\frac{\beta-1}{v^{2}}+\frac{\beta}{v}\right.\right. \\
\left.\left.+S\left\{-\beta^{2} t^{3}+p \int_{0}^{t} \underline{x}^{2}(s, \beta) d s\right\}\right)\right\}, \\
\bar{x}(t, \beta) \\
=S^{-1}\left\{v ^ { 2 } \left(\frac{1-\beta}{v^{2}}+\frac{2-\beta}{v}\right.\right. \\
\left.\left.+S\left\{-(2-\beta)^{2} t^{3}+p \int_{0}^{t} \bar{x}^{2}(s, \beta) d s\right\}\right)\right\} .
\end{array}\right.
$$

The solution (Figure 3 ) in powers of $p$ is represented as

$$
\begin{aligned}
& p^{0}:\left\{\begin{array}{l}
\underline{x}_{0}(t, \beta)=(\beta-1)+\beta t-\beta^{2} \frac{t^{5}}{20} \beta^{2} \\
\bar{x}_{0}(t, \beta)=(1-\beta)+t(2-\beta)-(2-\beta)^{2} \frac{t^{5}}{20},
\end{array}\right. \\
& \left(\underline{x}_{1}(t, \beta)=\frac{t^{3}}{6}(\beta-1)+\frac{t^{4}}{12} \beta(\beta-1)+\frac{t^{5}}{60} \beta^{2}\right. \\
& +\frac{t^{13}}{686400} \beta^{4}-\frac{t^{9}}{3024} \beta^{2}-\frac{t^{8}}{3360} \beta^{2}(\beta-1), \\
& p^{1}:\left\{\begin{aligned}
\bar{x}_{1}(t, \beta)= & \frac{t^{3}}{6}(1-\beta)+\frac{t^{4}}{12}(2-\beta)(1-\beta)+\frac{t^{5}}{60}(2-\beta)^{2} \\
& +\frac{t^{13}}{686400}(2-\beta)^{4}-\frac{t^{9}}{3024}(2-\beta)^{2} \\
& -\frac{t^{8}}{3360}(2-\beta)^{2}(1-\beta), \\
\underline{y}(t, \beta)= & t(\beta-1)+\frac{t^{2}}{2} \beta-\frac{t^{5}}{20} \beta^{2}+\frac{t^{5}}{120}(\beta-1)^{2} \\
& +\frac{t^{7}}{840} \beta^{2}+\frac{t^{13}}{1716} \beta^{4}+\frac{t^{7}}{840} \beta(\beta-1)
\end{aligned}\right.
\end{aligned}
$$

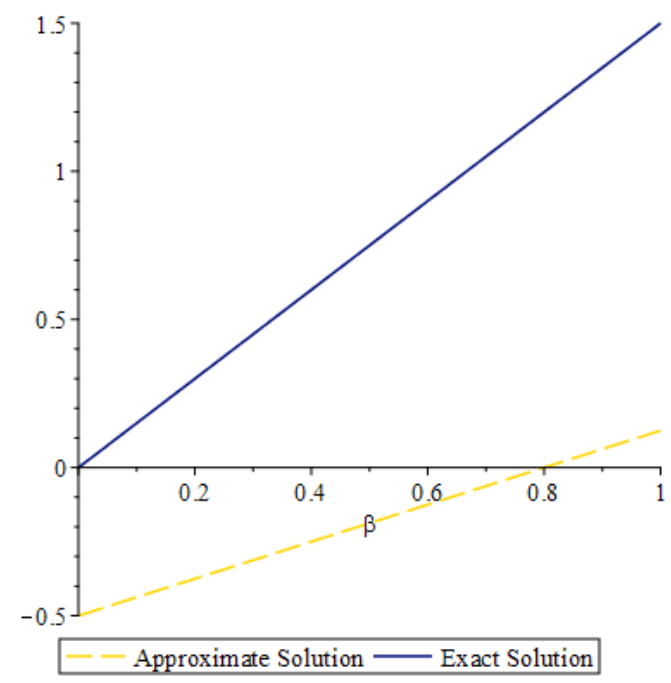

(a)

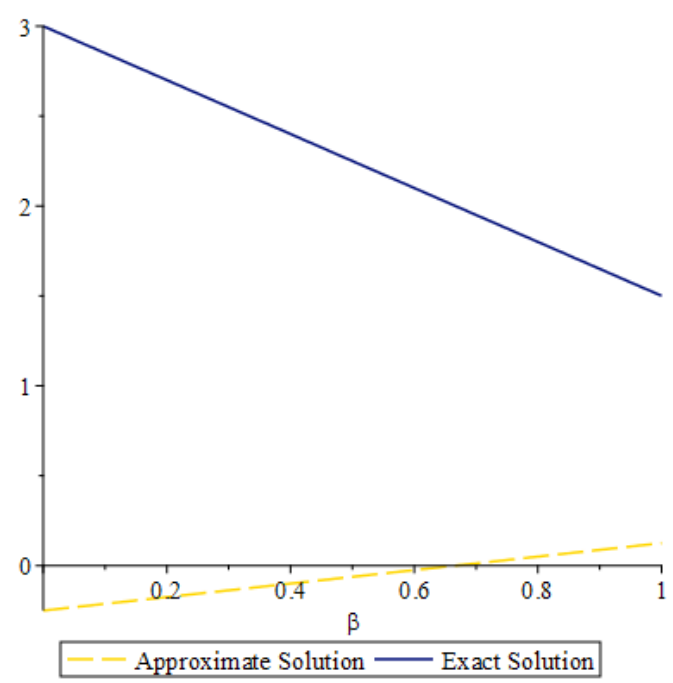

(b)

Figure 3. Comparison of approximate solution (33) and exact solution of (a) $\underline{y}$ and (b) $\bar{y}$ at $t=1$.

$$
\left\{\begin{aligned}
& -\frac{t^{10}}{14400} \beta^{3}-\frac{t^{9}}{10080} \beta^{2}(\beta-1), \\
\bar{y}(t, \beta)= & t(1-\beta)+\frac{t^{2}}{2}(2-\beta)-\frac{t^{5}}{20}(2-\beta)^{2} \\
& +\frac{t^{5}}{120}(1-\beta)^{2}+\frac{t^{7}}{840}(2-\beta)^{2}+\frac{t^{13}}{1716}(2-\beta)^{4} \\
& +\frac{t^{7}}{840}(2-\beta)(1-\beta)-\frac{t^{10}}{14400}(2-\beta)^{3} \\
& -\frac{t^{9}}{10080}(2-\beta)^{2}(1-\beta) .
\end{aligned}\right.
$$

Problem 4.4. Consider the nonlinear IDE of the second 
kind [11]

$$
y^{\prime}(t, \beta)=g(t, \beta)+\int_{0}^{1} \frac{s^{2}}{2} y^{2}(s, \beta) d s,
$$

with the initial condition $y(0, \beta)=(0,0)$, where $0 \leq t, s \leq 1$, $0 \leq \beta \leq 1, \lambda=1, K(t, s)=\frac{s^{2}}{2} \cdot g(t, \beta)=\left(\left(\beta-\frac{\beta^{2}}{8}\right),(12-\right.$ $\left.\left.4 \beta-\beta^{2}\right)\right)$, The exact solution is $(\beta t,(2-\beta) t)$. According to the detail procedure described above, we have

$$
\left\{\begin{array}{l}
\underline{x}(t, \beta)=S\left\{v\left(S\left\{\left(\beta-\frac{\beta^{2}}{8}\right)+p \int_{0}^{1} \frac{s^{2}}{2} x^{2}(s, \beta) d s\right\}\right)\right\}, \\
\bar{x}(t, \beta)=S\left\{v\left(S\left\{\left(\beta-\frac{\beta^{2}}{8}\right)+p \int_{0}^{1} \frac{s^{2}}{2} \bar{x}^{2}(s, \beta) d s\right\}\right)\right\} .
\end{array}\right.
$$

The solution components are

$$
\begin{aligned}
& p^{0}:\left\{\begin{array}{l}
\underline{x}_{0}(t, \beta)=\frac{-t}{8}(-8+\beta) \beta, \\
\bar{x}_{0}(t, \beta)=\frac{-t}{8}\left(-12+4 \beta+\beta^{2}\right),
\end{array}\right. \\
& p^{1}:\left\{\begin{array}{l}
\underline{x}_{1}(t, \beta)=\frac{t}{640}(-8+\beta)^{2} \beta^{2}, \\
\bar{x}_{1}(t, \beta)=\frac{t}{640}\left(-12+4 \beta+\beta^{2}\right)^{2},
\end{array}\right. \\
& p^{2}:\left\{\begin{array}{l}
\underline{x}_{2}(t, \beta)=\frac{t}{4096000}(-8+\beta)^{4} \beta^{4}, \\
\bar{x}_{2}(t, \beta)=\frac{t}{4096000}\left(-12+4 \beta+\beta^{2}\right)^{4},
\end{array}\right.
\end{aligned}
$$

and so on. The solution (Figure 4) is represented by utilizing the above iterations as

$$
\left\{\begin{aligned}
\underline{y}(t, \beta)= & \frac{-t}{8}(-8-\beta) \beta+\frac{t}{640}(-8-\beta)^{2} \beta^{2} \\
& +\frac{t}{4096000}(-8-\beta)^{4} \beta^{4} \\
\bar{y}(t, \beta)= & \frac{-t}{8}\left(-12+4 \beta+\beta^{2}\right) \\
& +\frac{t}{640}\left(-12+4 \beta+\beta^{2}\right)^{2} \frac{t}{4096000}\left(-12+4 \beta+\beta^{2}\right)^{4}
\end{aligned}\right.
$$

Problem 4.5. Finally, consider the nonlinear fuzzy VIDE of the first order as [11]

$$
y^{\prime}(t, \beta)=g(t, \beta)+\int_{0}^{t} y^{2}(s, \beta) d s,
$$

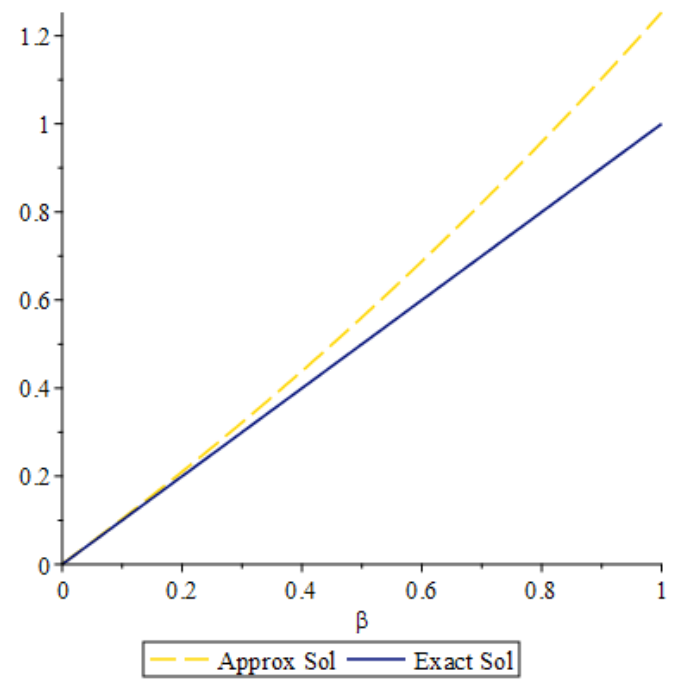

(a)

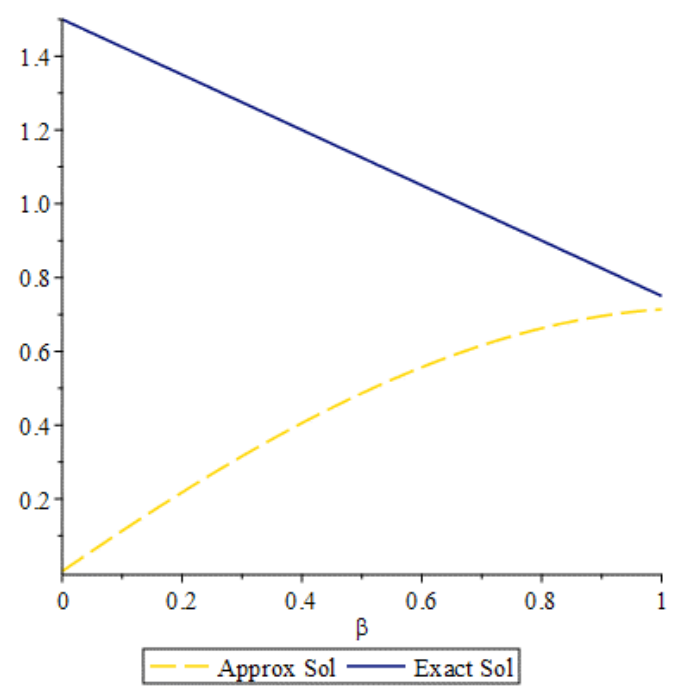

(b)

Figure 4. Comparison of approximate solution (39) and exact solution of (a) $\underline{y}$ and (b) $\bar{y}$ at $t=1$.

with initial condition $y(0, \beta)=(0,0)$, where $0 \leq t, s \leq 1$, $0 \leq \beta \leq 1, \lambda=1, K(t, s)=1$, and

$$
g(t, \beta)=\left(\begin{array}{l}
\left(-\frac{1}{5}\left(\beta^{4}+2 \beta^{3}+\beta^{2}\right) t^{5}+2\left(\beta^{2}+\beta\right) t\right), \\
\left(-\frac{1}{5}\left(16-8 \beta^{3}-8 \beta+\beta^{6}+2 \beta^{4}+\beta^{2}\right) t^{5}\right. \\
\left.+2\left(4-\beta^{3}+\beta\right) t\right),
\end{array}\right) .
$$

The exact solution of Eq. (40) is given as

$$
y(t, \beta)=\left(\left(\beta^{2}+\beta\right) t^{2},\left(4-\beta^{3}+\beta\right) t^{2}\right) .
$$


We construct the homotopy as

$$
\left\{\begin{aligned}
H(x, p, \beta)= & \underline{x^{\prime}}(t, \beta)+\frac{1}{5}\left(\beta^{4}+2 \beta^{3}+\beta^{2}\right) t^{5} \\
& -2\left(\beta^{2}+\beta\right) t-p \int_{0}^{t} \underline{x}^{2}(s, \beta) d s=0 \\
H(\bar{x}, p, \beta)= & \overline{x^{\prime}}(t, \beta)+\frac{1}{5}\left(16-8 \beta^{3}-8 \beta+\beta^{6}+2 \beta^{4}+\beta^{2}\right) t^{5} \\
& -2\left(4-\beta^{3}+\beta\right) t-p \int_{0}^{t} \bar{x}^{2}(s, \beta) d s=0 .
\end{aligned}\right.
$$

According to the procedure described above, we have

$$
\left\{\begin{array}{l}
\underline{x}(t, \beta) \\
=S^{-1}\left\{v \left(S \left\{-\frac{1}{5}\left(\beta^{4}+2 \beta^{3}+\beta^{2}\right) t^{5}+2\left(\beta^{2}+\beta\right) t\right.\right.\right. \\
\left.\left.\left.\quad+p \int_{0}^{t} \underline{x}^{2}(s, \beta) d s\right\}\right)\right\}, \\
\bar{x}(t, \beta) \\
=S^{-1}\left\{v \left(S \left\{-\frac{1}{5}\left(16-8 \beta^{3}-8 \beta+\beta^{6}+2 \beta^{4}+\beta^{2}\right) t^{5}\right.\right.\right. \\
\left.\left.\left.\quad+2\left(4-\beta^{3}+\beta\right) t+p \int_{0}^{t} \bar{x}^{2}(s, \beta) d s\right\}\right)\right\}
\end{array}\right.
$$

Comparing the powers of parameter $\mathrm{p}$ in Eq. 42, we have

$$
p^{0}:\left\{\begin{aligned}
\underline{x}_{0}(t, \beta) & =\left(\beta^{2}+\beta\right) t^{2}-\frac{1}{30}\left(\beta^{4}+2 \beta^{3}+\beta^{2}\right) t^{6}, \\
\overline{x_{0}}(t, \beta) & =\left(4-\beta^{3}+\beta\right) t^{2} \\
& -\frac{1}{30}\left(16-8 \beta^{3}-8 \beta+\beta^{6}+2 \beta^{4}+\beta^{2}\right) t^{6},
\end{aligned}\right.
$$

$$
p^{1}:\left\{\begin{aligned}
\underline{x}_{1}(t, \beta)= & \left(\beta^{2}+\beta\right)^{2} \frac{t^{6}}{30}+\left(\beta^{4}+2 \beta^{3}+\beta^{2}\right)^{2} \frac{t^{14}}{163800}, \\
& -\left(\beta^{2}+\beta\right)\left(\beta^{4}+2 \beta^{3}+\beta^{2}\right) \frac{t^{10}}{1350}, \\
\overline{x_{1}}(t, \beta)= & \left(4-\beta^{3}+\beta\right)^{2} \frac{t^{6}}{30} \\
& +\left(16-8 \beta^{3}-8 \beta+\beta^{6}+2 \beta^{4}+\beta^{2}\right)^{2} \frac{t^{14}}{163800}, \\
& -\left(4-\beta^{3}+\beta\right)\left(16-8 \beta^{3}-8 \beta+\beta^{6}\right. \\
& \left.+2 \beta^{4}+\beta^{2}\right) \frac{t^{10}}{1350},
\end{aligned}\right.
$$

and so on. Utilizing the above results and we get series solution (Figure 5) as

$$
\left\{\begin{aligned}
\underline{y}(t, \beta) & =\left(\beta^{2}+\beta\right) t^{2}-\frac{1}{30}\left(\beta^{4}+2 \beta^{3}+\beta^{2}\right) t^{6} \\
& +\frac{1}{30}\left(\beta^{2}+\beta\right)^{2} t^{6}+\left(\beta^{4}+2 \beta^{3}+\beta^{2}\right)^{2} \frac{t^{14}}{163800} \\
& -\left(\beta^{2}+\beta\right)\left(\beta^{4}+2 \beta^{3}+\beta^{2}\right) \frac{t^{10}}{1350}, \\
\bar{y}(t, \beta) & =\left(4-\beta^{3}+\beta\right) t^{2} \\
& -\frac{1}{30}\left(16-8 \beta^{3}-8 \beta+\beta^{6}+2 \beta^{4}+\beta^{2}\right) t^{6} \\
& +\left(4-\beta^{3}+\beta\right)^{2} \frac{t^{6}}{30} \\
& +\left(16-8 \beta^{3}-8 \beta+\beta^{6}+2 \beta^{4}+\beta^{2}\right)^{2} \overline{163800} \\
& -\left(4-\beta^{3}+\beta\right)\left(16-8 \beta^{3}-8 \beta+\beta^{6}\right. \\
& \left.+2 \beta^{4}+\beta^{2}\right) \frac{t^{10}}{1350} .
\end{aligned}\right.
$$

\section{Conclusion}

In this study, we investigated the HPSTM in order to solve nonlinear FIDEs based on the parametric form of fuzzy numbers. The solutions obtained by these fuzzy differential equations are considered to be controllers in applications. This method proved its effectiveness and reliability in solving uncertain types of equations by providing the best approximate solutions. The numerical outcomes obtained using the proposed technique are comparable to the exact solutions. Thus, the work can be extended to higher-order mixed-type FIDEs in more variables.

\section{Conflict of Interest}

The authors have no conflict of interest.

\section{References}

[1] L. A. Zadeh, "Fuzzy sets," Information and Control, vol. 8, no. 3, pp. 338-353, 1965. https://doi.org/10.1016/S00199958(65)90241-X

[2] L. A. Zadeh, "A fuzzy-algorithmic approach to the definition of complex or imprecise concepts," International Journal of Man-machine studies, vol. 8, no. 3, pp. 249-291, 1976. https://doi.org/10.1016/S0020-7373(76)80001-6 


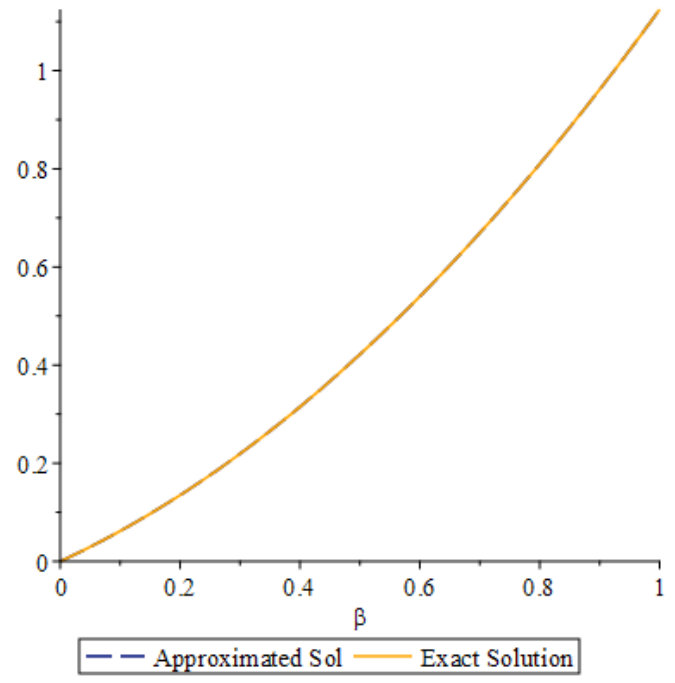

(a)

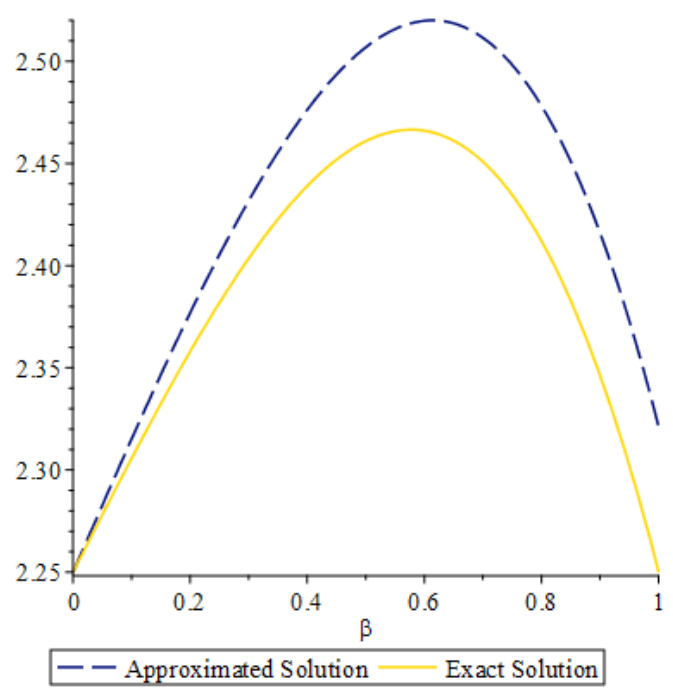

(b)

Figure 5. Comparison of approximate solution (45) and exact solution of (a) $y$ and (b) $\bar{y}$ at $t=1$.

[3] D. Dubois and H. Prade, "Towards fuzzy differential calculus part 1: Integration of fuzzy mappings," Fuzzy Sets and Systems, vol. 8, no. 1, pp. 1-17, 1982. https: //doi.org/10.1016/0165-0114(82)90025-2

[4] K. Shah, A. R. Seadawy, and M. Arfan, "Evaluation of one dimensional fuzzy fractional partial differential equations," Alexandria Engineering Journal, vol. 59, no. 5, pp. 33473353, 2020. https://doi.org/10.1016/j.aej.2020.05.003

[5] N. Ahmad, A. Ullah, A. Ullah, S. Ahmad, K. Shah, and I. Ahmad, "On analysis of the fuzzy fractional order VolterraFredholm integro-differential equation," Alexandria En- gineering Journal, vol. 60, no. 1, pp. 1827-1838, 2021. https://doi.org/10.1016/j.aej.2020.11.031

[6] A. Ullah, Z. Ullah, T. Abdelwajid, Z. Hammouch, and K. Shah, "A hybrid method for solving fuzzy Volterra integral equations of separable type kernels," Journal of King Saud University-Science, vol. 33, no. 1, article no. 101246, 2021. https://doi.org/10.1016/j.jksus.2020.101246

[7] M. U. Rahman, M. Arfan, K. Shah, and J. F. GomezAguilar, "Investigating a nonlinear dynamical model of COVID-19 disease under fuzzy caputo, random and ABC fractional order derivative," Chaos, Solitons \& Fractals, vol. 140, article no. 110232, 2020. https://doi.org/10.1016/ j.chaos.2020.110232

[8] Z. Ulla, A. Ullah, K. Shah, and D. Baleanu, "Computation of semi-analytical solutions of fuzzy nonlinear integral equations," Advances in Difference Equations, vol. 2020, article no. 542, 2020. https://doi.org/10.1186/s13662-02002989-z

[9] J. Ahmad and H. Nosher, "Solution of different types of fuzzy integro-differential equations via Laplace homotopy perturbation method," Journal of Science and Arts, vol. 17, no. 1, pp. 5-22, 2017.

[10] A. A. Hamoud and K. P. Ghadle, "Modified Adomian decomposition method for solving fuzzy Volterra-Fredholm integral equation," Journal of the Indian Mathematical Society, vol. 85, no. 1-2, pp. 53-69, 2018. https://doi.org/ $10.18311 /$ jims/2018/16260

[11] L. Hooshangian, "Nonlinear fuzzy Volterra integrodifferential equation of N-th order: analytic solution and existence and uniqueness of solution," International Journal of Industrial Mathematics, vol. 11, no. 1, pp. 43-54, 2019.

[12] Y. C. Kwun, C. W. Han, S. Y. Kim, and J. S. Park, "Existence and uniqueness of fuzzy solutions for the nonlinear fuzzy integro-differential equation on $E_{N}^{n}$," International Journal of Fuzzy Logic and Intelligent Systems, vol. 4, no. 1, pp. 40-44, 2004. https://doi.org/10.5391/ijfis.2004.4.1. 040

[13] Y. C. Kwun, J. S. Kim, and J. H. Park, "Existence of solutions for the impulsive semilinear fuzzy intergrodifferential equations with nonlocal conditions and forcing term with memory in n-dimensional fuzzy vector 
space $\left(E_{N}^{n}, d_{\varepsilon}\right)$," International Journal of Fuzzy Logic and Intelligent Systems, vol. 11, no. 1, pp. 25-32, 2011. https://doi.org/10.5391/IJFIS.2011.11.1.025

[14] V. Padmapriya, M. Kaliyappan, and A. Manivannan, "Numerical solutions of fuzzy fractional delay differential equations," International Journal of Fuzzy Logic and Intelligent Systems, vol. 20, no. 3, pp. 247-254, 2020. http://doi.org/10.5391/IJFIS.2020.20.3.247

[15] J. H. He, "A coupling method of a homotopy technique and a perturbation technique for non-linear problems," International Journal of Nonlinear Mechanics, vol. 35, no. 1, pp. 37-43, 2000. https://doi.org/10.1016/S00207462(98)00085-7

[16] J. H. He, "Homotopy perturbation technique," Computer Methods in Applied Mechanics and Engineering, vol. 178, no. 3-4, pp. 257-262, 1999. https://doi.org/10.1016/S00457825(99)00018-3

[17] M. A. Rana, A. M. Siddiqui, Q. K. Ghori, and R. Qamar, "Application of He's homotopy perturbation method to Sumudu transform," International Journal of Nonlinear Sciences and Numerical Simulation, vol. 8, no. 2, pp. 185190, 2007. https://doi.org/10.1515/IJNSNS.2007.8.2.185

[18] G. K. Watugala, "Sumudu transform: a new integral transform to solve differential equations and control engineering problems," International Journal of Mathematical Education in Science and Technology, vol. 24, pp. 35-43, 1993. https://doi.org/10.1080/0020739930240105

[19] M. Mosleh and M. Otadi, "Existence of solution of nonlinear fuzzy Fredholm integro-differential equations," Fuzzy Information and Engineering, vol. 8, no. 1, pp, 17-30, 2016. https://doi.org/10.1016/j.fiae.2016.03.002

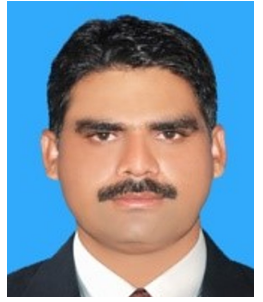

Jamshad Ahmad is currently working as an Assistant Professor in the Department of Mathematics, University of Gujrat, Pakistan. He received his Ph.D. degree in 2015 from HITEC University, Taxila, Pakistan. His research interests include computational and applied mathematics.

E-mail:

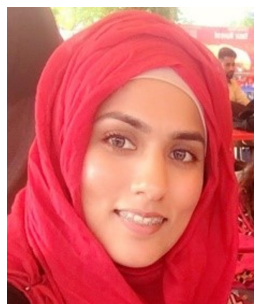

Angbeen Iqbal received his Master of Philosophy in Mathematics in 2019 from the University of Gujrat, Pakistan. She did her Bachelor of Science degree in Mathematics in 2017 from the University of Gujrat, Pakistan. Her research interests include fuzzy differential equations and fuzzy fractional differential equations.

E-mail:

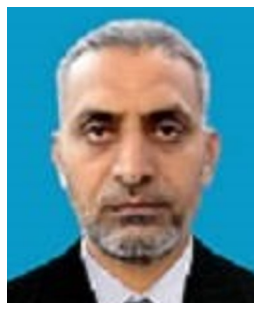

Qazi Mahmood Ul-Hassan is currently working as an Associate Professor in the Department of Mathematics, University of Wah, Wah Cantt. Pakistan. He received his Ph.D. from HITEC University, Taxila Cantt, Pakistan. His research interests include computational and applied mathematics.

E-mail: 